\title{
Editorial Note: Efficient Multimedia Processing Methods and Applications
}

\author{
Published online: 14 September 2018 \\ C Springer Science+Business Media, LLC, part of Springer Nature 2018
}

Multimedia Tools and Applications gratefully acknowledges the editorial work of the scholars listed below on the special issue entitled "Efficient Multimedia Processing Methods and Applications."

Of 40 papers submitted to this issue, 20 were eventually accepted after a stringent peerreview process.

\author{
Weiqing Min (Corresponding Guest Editor) \\ Institute of Computing Technology, Chinese Academy of Sciences, Beijing, China \\ minweiqing@ict.ac.cn
}

Bing-kun Bao

Institute of Automation, Chinese Academy of Sciences, Beijing, China

bkbao@nlpr.ia.ac.cn

Qianqian Xu

Institute of Information Engineering, Chinese Academy of Sciences, Beijing, China xuqianqian@iie.ac.cn

Luis Herranz

Computer Vision Center, Barcelona, Spain

lherranz@cvc.uab.es

\section{Shuqiang Jiang}

Institute of Computing Technology, Chinese Academy of Sciences, Beijing, China sqjiang@ict.ac.cn

Publisher's Note Springer Nature remains neutral with regard to jurisdictional claims in published maps and institutional affiliations. 